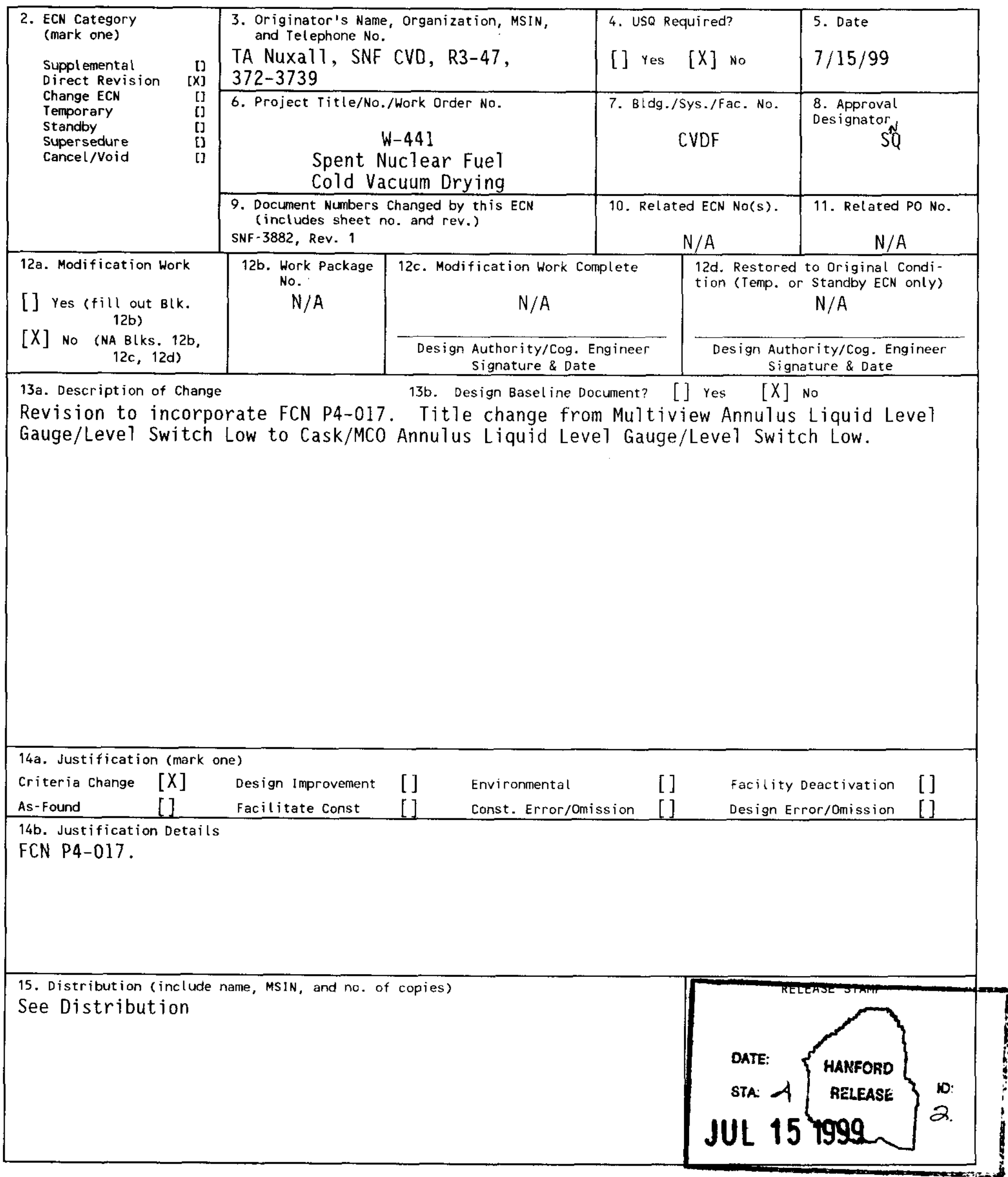




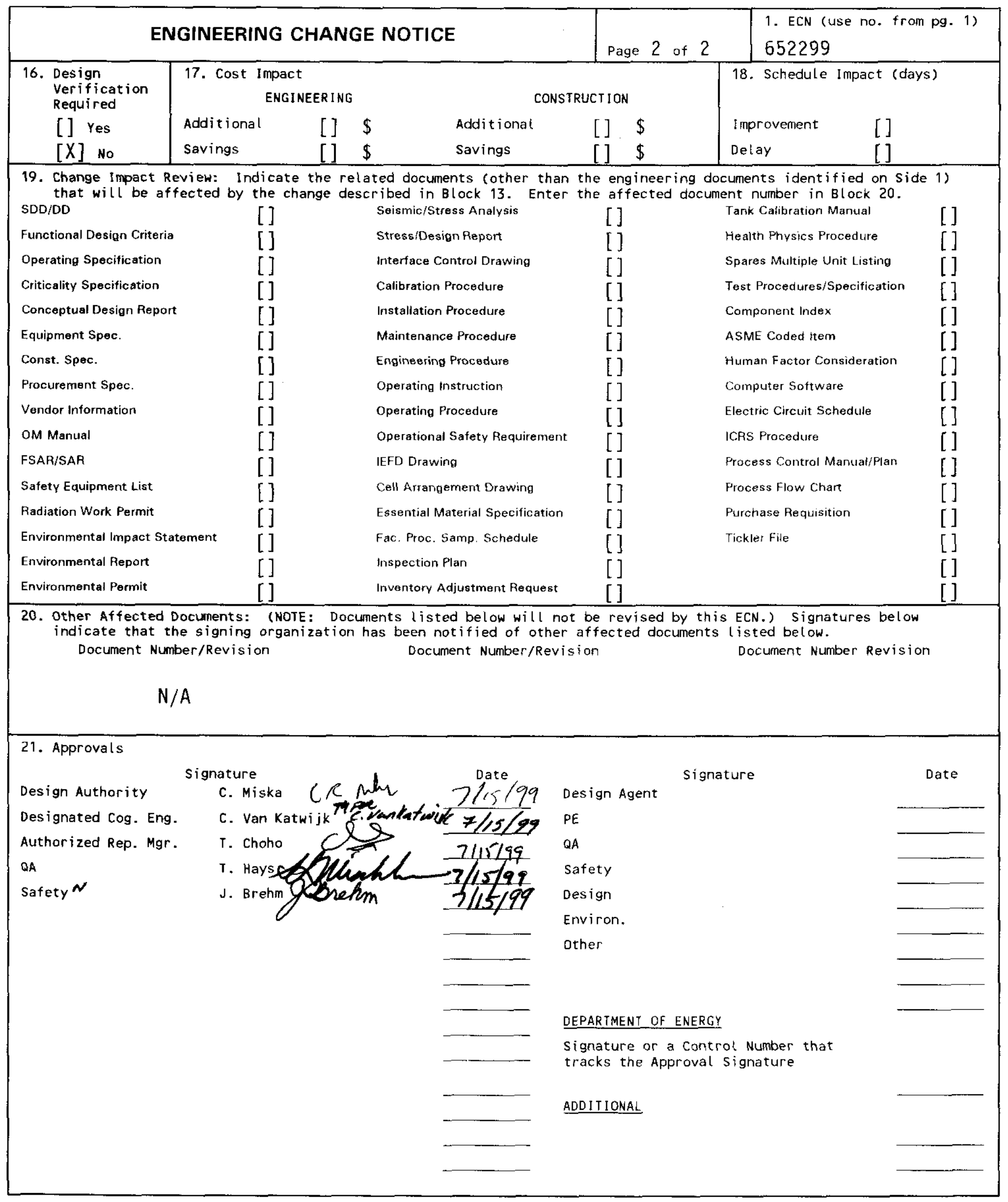




\section{CASK/MCO ANNULUS LIQUID LEVEL GAUGE/LEVEL SWITCH LOW}

Carl Van Katwijk

Numatec Hanford, Richland, WA 99352

U.S. Department of Energy Contract DE-AC06-96RL13200

EDT/ECN: 652299

Org Code: $2 \mathrm{G} 300$

B\&R Code: 39EW40400
UC: 620

Charge Code: 105559/A000

Total Pages: 14

Key Words: Multiview Annulus Liquid Level Gauge/Level Switch Low

Abstract: Multiview Annulus Liquid Level Gauge/Level Switch Low

TRADEMARK DISCLAIMER. Reference herein to any specific commercial prodict, process. or service by trade name, trademark. manufacturer, or otherwise, does not necessarily constitute or imply its endorsement, recommendation. or favoring by the I Inited Stales Government or any agency thereof or its contractors or subcontractors.

Printed in the United States of America. To obtain copies of this document, contact: Document Control Services. P.O. Box 950, Mailstop H6-08, Richland WA 99352. Phone (509) 372-2420; Fax (509) 376-4989.

\section{Approved for Public Release}




\section{RECORD OF REVISION}

(2) Title

CASK/MCO ANNULUS LIQUID LEVEL GAUGE/LEVEL SWITCH LOW

Change Control Record

(3) Revision

$(7)$

$0 \quad$ EDT 626256

2
(4) Description of Change - Replace, Add, and Delete Pages

ECN 653776 , REVISION TO MEET SEL REV. 6

RS
ECN 652299, INCORPORATED CHANGES PER FCN P4-017. TITLE CHANGE TO DOCUMENT.
Authorized for Release

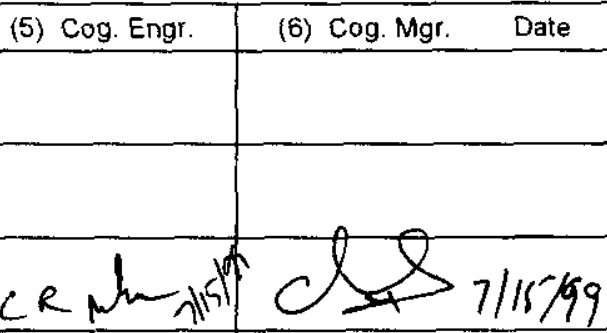

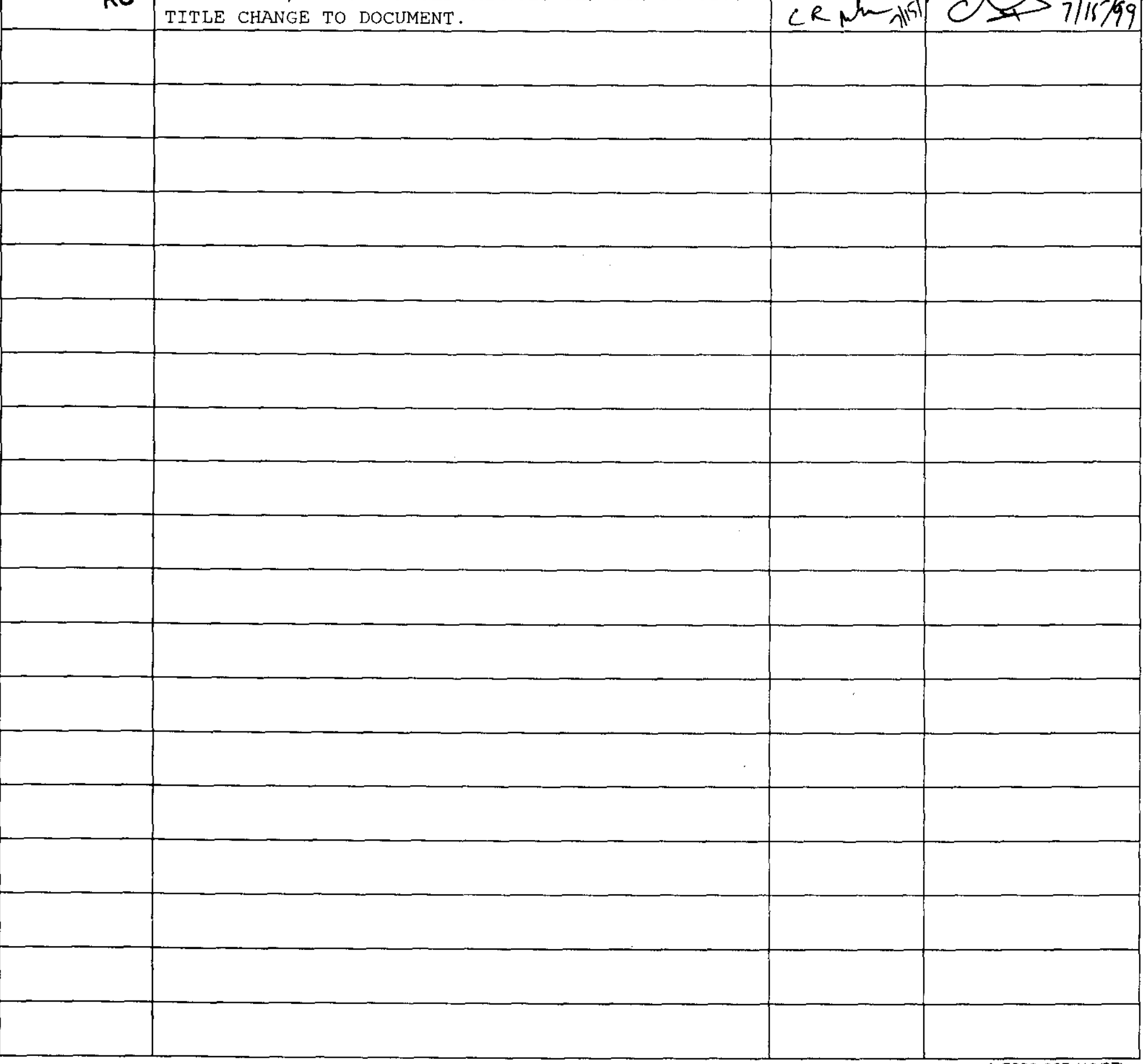




\begin{tabular}{|l|l|}
\hline Commercial Grade Item Upgrade Dedication Form & SNF-3882, Rev. 2 \\
\hline ECN No. NA CGI No. CGI-SNF-D-47-P4-007 & Page 1 of 12 \\
Title: CASK/MCO ANNULUS LIQUID LEVEL GAUGE/LEVEL SWITCH & \\
LOW & \\
\hline
\end{tabular}

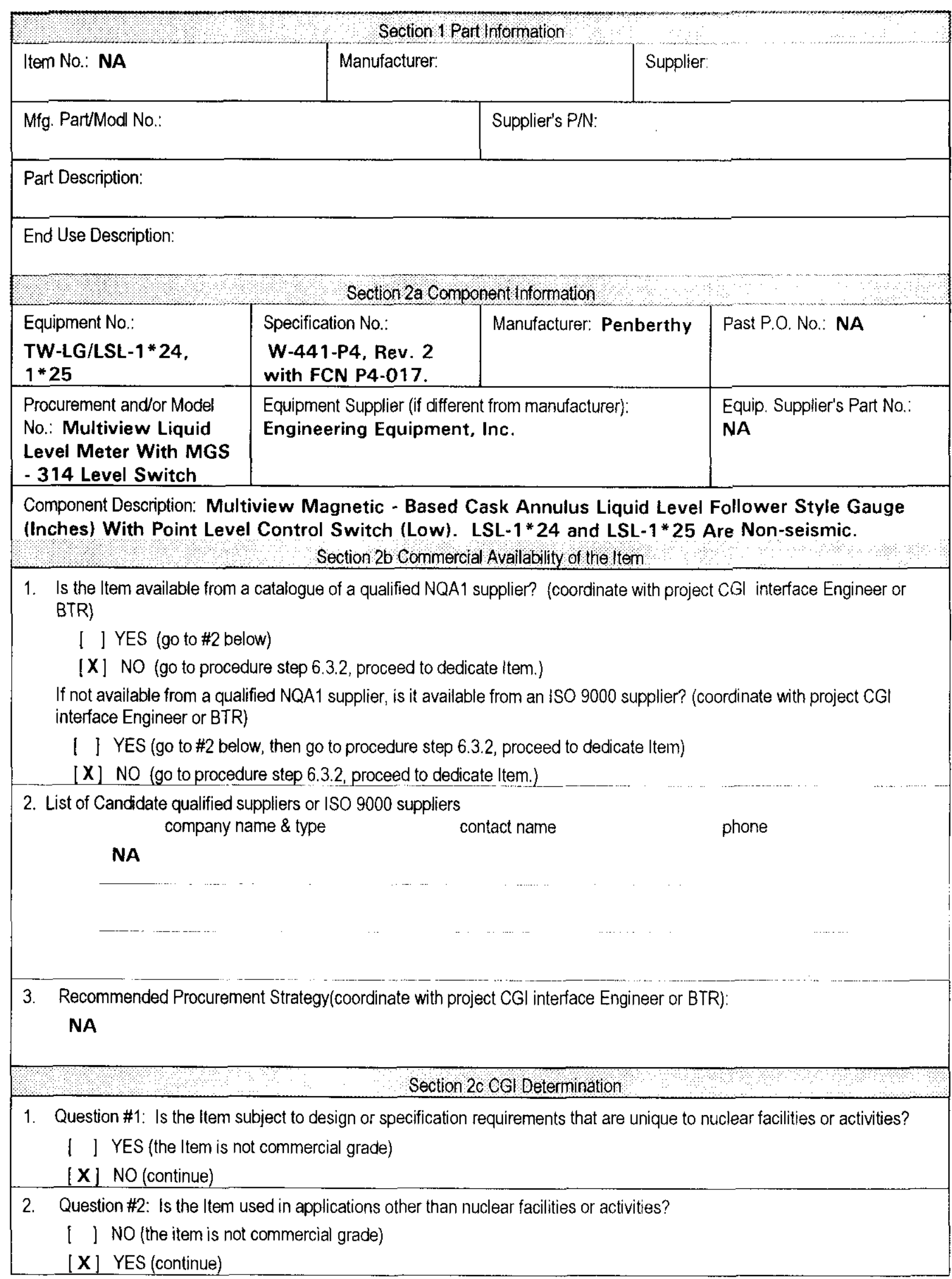




\begin{tabular}{|c|c|}
\hline Commercial Grade Item Upgrade Dedication Form & SNI-3882, Rev. 2 \\
\hline $\begin{array}{l}\text { ECN No. } \frac{\text { NA }}{\text { CGI No. CGI-SNF-D-47-P4-007 }} \\
\text { Title: } \text { CASK/MCO ANNULUS LIQUID LEVEL GAUGE/LEVEL SWITCH } \\
\text { LOW }\end{array}$ & Page 2 of 12 \\
\hline
\end{tabular}

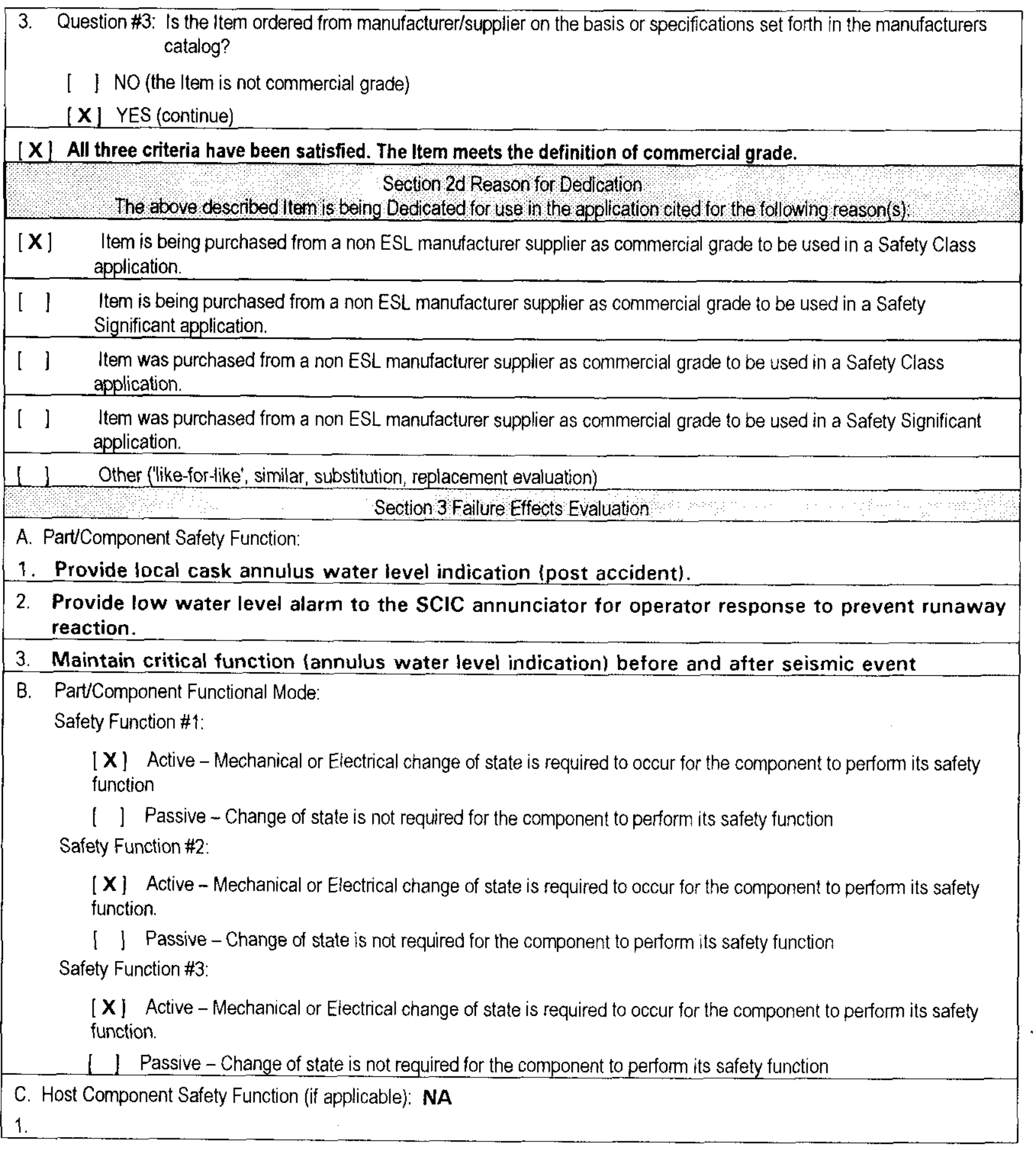


D. Failure Mode(s) and the effects on component or system safety function (see Worksheet 1):

1. Failure of or stuck follower - loss of reading and alarm.

2. Failure of low level switch contacts to make or break - loss of alarm to SCIC.

Section 4 Environmental \& Natural Phenomena Hazand Design

$$
\begin{aligned}
& \text { Yes [ ] } \\
& \text { No [X] } \\
& \text { Phenomena } \\
& \text { Yes [ } \mathbf{X}] \\
& \text { No [ ] }
\end{aligned}
$$

Environmental Qualification Required

Environmental Condition B

Natural Phenomena Hazard (NPH) Design Required

HNF-PRO-97, Rev. 0

W-441-P4, Rev. 2 with FCN P4-017
If yes: Environmental Qualification Requirements

Limiting Environmental Conditions:

Required Safety Functions:

Qualification Period:

If yes: NPH Design Requirements

Performance Category: LG-1* 24,-1*25 PC-3

LSL $-1 * 24,-1$ * 25 PC -1

NPH Design Req'ts.: Seismic Condition A - LG-1*24, $1 * 25$ only. N/A for LSL- $1 * 24,-1 * 25$

Required Safety Functions: Cask annulus water level indication; Low Level Alarm (alarm not required for seismic event)

Section 5 Component runctional Classification

[X] Safety Class (SC) [ ] General Service [ ] Safety Significant (SS)

If part/component classification is different from host component/system, document basis.

Section 6 (Resenved)

Section 7 (Reserved)

Section 8 References (for Functional Classification)

National Codes/Standards:

IEEE 344, IEEE 379, IEEE Safety Analysis Report (SAR): HNF. SD-SNF-SAR-002, Rev. 4 A

Drawings: H-1-82161, Rev. 2 603, IEEE 627 HNF-SD-SNF-SEL-002, Rev. 6A

Vendor Manual/Manufacturer/Supplier Information: Penberthy Multiview Manual, Application Report 4771

Other: NA 
Commercial Grade Item Upgrade Dedication Form

ECN No. NA CGI No. CGI-SNF-D-47-P4-007

Title: CASK/MCO ANNULUS LIQUID LEVEL GAUGE/LEVEL SWITCH LOW

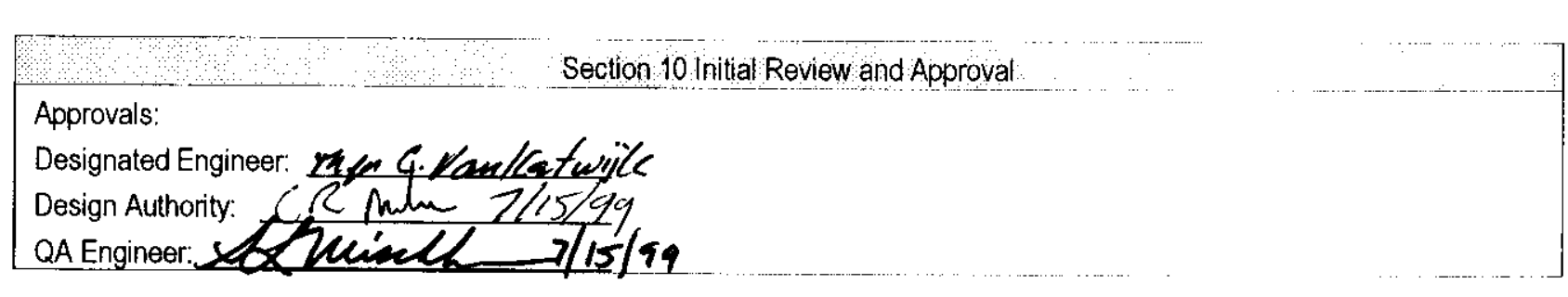

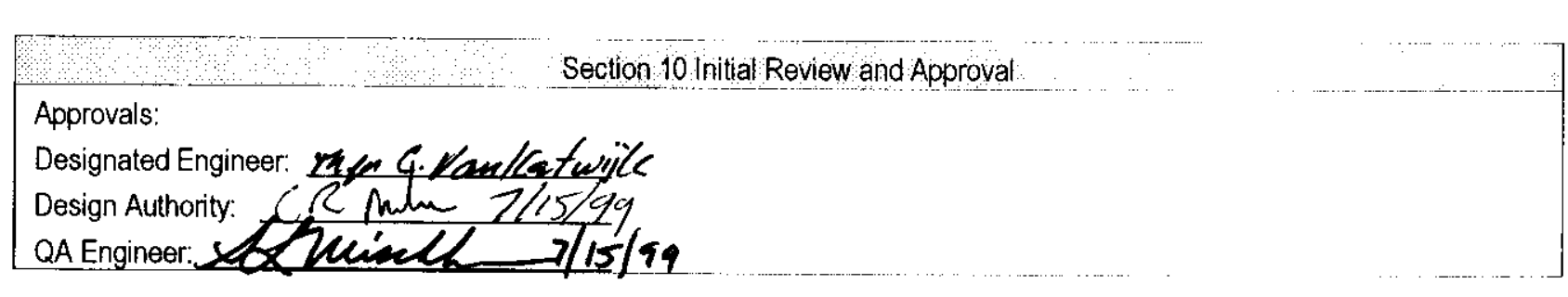

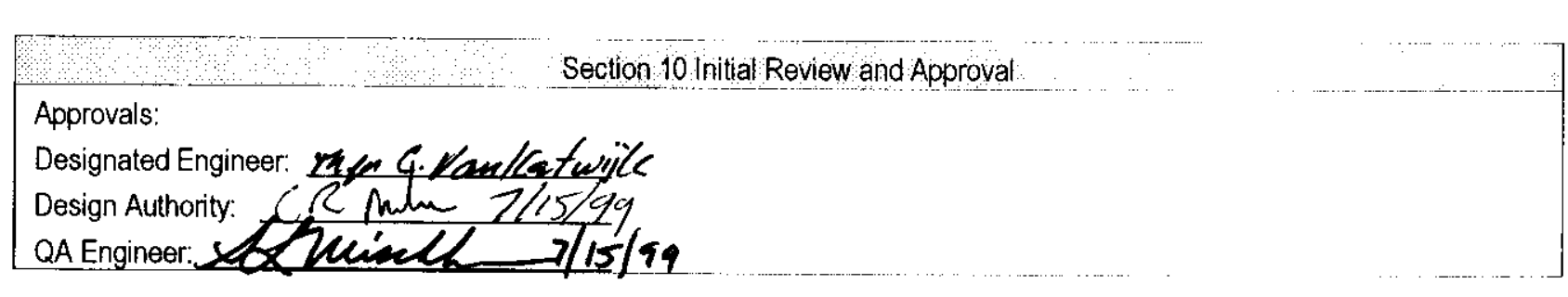

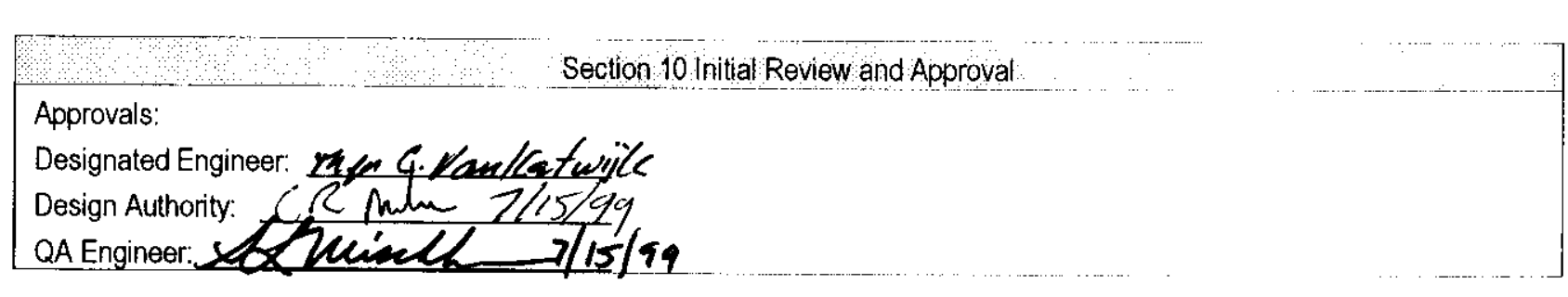

SNF-3882. Rev. 2

Page 5 of 12 


\begin{tabular}{|c|c|c|}
\hline \multicolumn{3}{|c|}{$\begin{array}{l}\text { WORKSHEET } 1 \\
\text { DETERMINATION OF FAILURE MECHANISMS/MODES } \\
\text { SECTION } 1\end{array}$} \\
\hline $\begin{array}{l}\text { Typical Failure } \\
\text { Mechanisms }\end{array}$ & Definition & $\begin{array}{l}\text { Applicable to Component } \\
\text { under Evaluation }\end{array}$ \\
\hline Fracture & $\begin{array}{l}\text { Separation of a solid accompanied by little or no } \\
\text { macroscopic plastic deformation. }\end{array}$ & $\begin{array}{l}\text { Yes [ ] No [ } \mathrm{X} \text { ]; If Yes, indicate failure } \\
\text { Mode }\end{array}$ \\
\hline Corrosion & $\begin{array}{l}\text { The gradual deterioration of a material due to } \\
\text { chemical or electrochemical reactions, such as } \\
\text { oxidation, between the material and its environment. }\end{array}$ & $\begin{array}{l}\text { Yes [ ] No [X]; If Yes, indicate failure } \\
\text { Mode }\end{array}$ \\
\hline Erosion & $\begin{array}{l}\text { Destruction of materials by the abrasive action of } \\
\text { moving fluids, usually accelerated by the presence } \\
\text { of solid particles carried with the fluid. }\end{array}$ & $\begin{array}{l}\text { Yes [ ] No [X]; If Yes, indicate failure } \\
\text { Mode }\end{array}$ \\
\hline Open Circuit & $\begin{array}{l}\text { An electrical circuit that is unintentionally broken so } \\
\text { that there is no complete path for current flow. }\end{array}$ & $\begin{array}{l}\text { Yes }[X] \text { No I ]; If Yes, indicate failure } \\
\text { Mode Switch Open/Short Circuit - } \\
\text { Loss of Alarm }\end{array}$ \\
\hline Short Circuit & $\begin{array}{l}\text { An abnormal connection by which an electrical } \\
\text { current is connected to ground, or to some } \\
\text { conducting body, resulting in excessive current flow. }\end{array}$ & $\begin{array}{l}\text { Yes }[X] \text { No [ ]; If Yes, indicate failure } \\
\text { Mode }\end{array}$ \\
\hline Blockage & $\begin{array}{l}\text { Clogging of a filtering medium resulting in the } \\
\text { inability to perform its purification function or } \\
\text { blockage of flow. }\end{array}$ & $\begin{array}{l}\text { Yes [ ] No [X]; If Yes, indicate failure } \\
\text { Mode_Above }\end{array}$ \\
\hline Seizure & $\begin{array}{l}\text { Binding of a normally moving item through excessive } \\
\text { pressure, temperature, friction, jamming. }\end{array}$ & $\begin{array}{l}\text { Yes [X] No [ ]; If Yes, indicate failure } \\
\text { Mode }\end{array}$ \\
\hline $\begin{array}{l}\text { Unacceptable } \\
\text { Vibration }\end{array}$ & $\begin{array}{l}\text { Mechanical oscillations produced are beyond the } \\
\text { defined permissible limits due to unbalancing, poor } \\
\text { support, or rotation at critical speeds. }\end{array}$ & $\begin{array}{l}\text { Yes [ I No [ } \mathbf{X} \text { ]; If Yes, indicate failure } \\
\text { Mode }\end{array}$ \\
\hline Loss of Properties & $\begin{array}{l}\text { A loss of mechanical and physical properties of a } \\
\text { material due to exposure to high temperatures, } \\
\text { radiation exposure. }\end{array}$ & $\begin{array}{l}\text { Yes [ }] \text { No }[\mathbf{X} \text { ]; If Yes, indicate failure } \\
\text { Mode }\end{array}$ \\
\hline Excess Strain & $\begin{array}{l}\text { Under the action of excessive external forces the } \\
\text { material of the part has been deformed or distorted. }\end{array}$ & $\begin{array}{l}\text { Yes [ I No [X I; If Yes, indicate failure } \\
\text { Mode }\end{array}$ \\
\hline Mechanical Creep & $\begin{array}{l}\text { From prolonged exposure to high temperature and } \\
\text { stress, the object will show a slow change in its } \\
\text { physical (shape and dimension) and mechanical }\end{array}$ & $\begin{array}{l}\text { Yes [ I No [X]; If Yes, indicate failure } \\
\text { Mode }\end{array}$ \\
\hline Ductile Fracture & $\begin{array}{l}\text { Fracture characterized by tearing of metal } \\
\text { accompanied by appreciable gross plastic } \\
\text { deformation. }\end{array}$ & $\begin{array}{l}\text { Yes [ ] No [ ]; If Yes, indicate failure } \\
\text { Mode }\end{array}$ \\
\hline 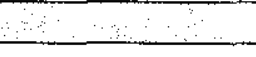 & ction 2 Additional Failure Modes Applicable to the C & onent Under Evaluation \\
\hline Failure c & Stuck Follower - Loss of Reading/Alarm & \\
\hline
\end{tabular}


Commercial Grade Item Upgrade Dedication Form

ECN No. NA CGI No. CGI-SNF-D-47-P4-007

Title: CASK/MCO ANNULUS LIQUID LEVEL GAUGE/LEVEL SWITCH

LOW

\section{CHECKLIST 1}

ACCEPTANCE METHOD 1

SPECIAL TEST/INSPECTION VERIFICATION

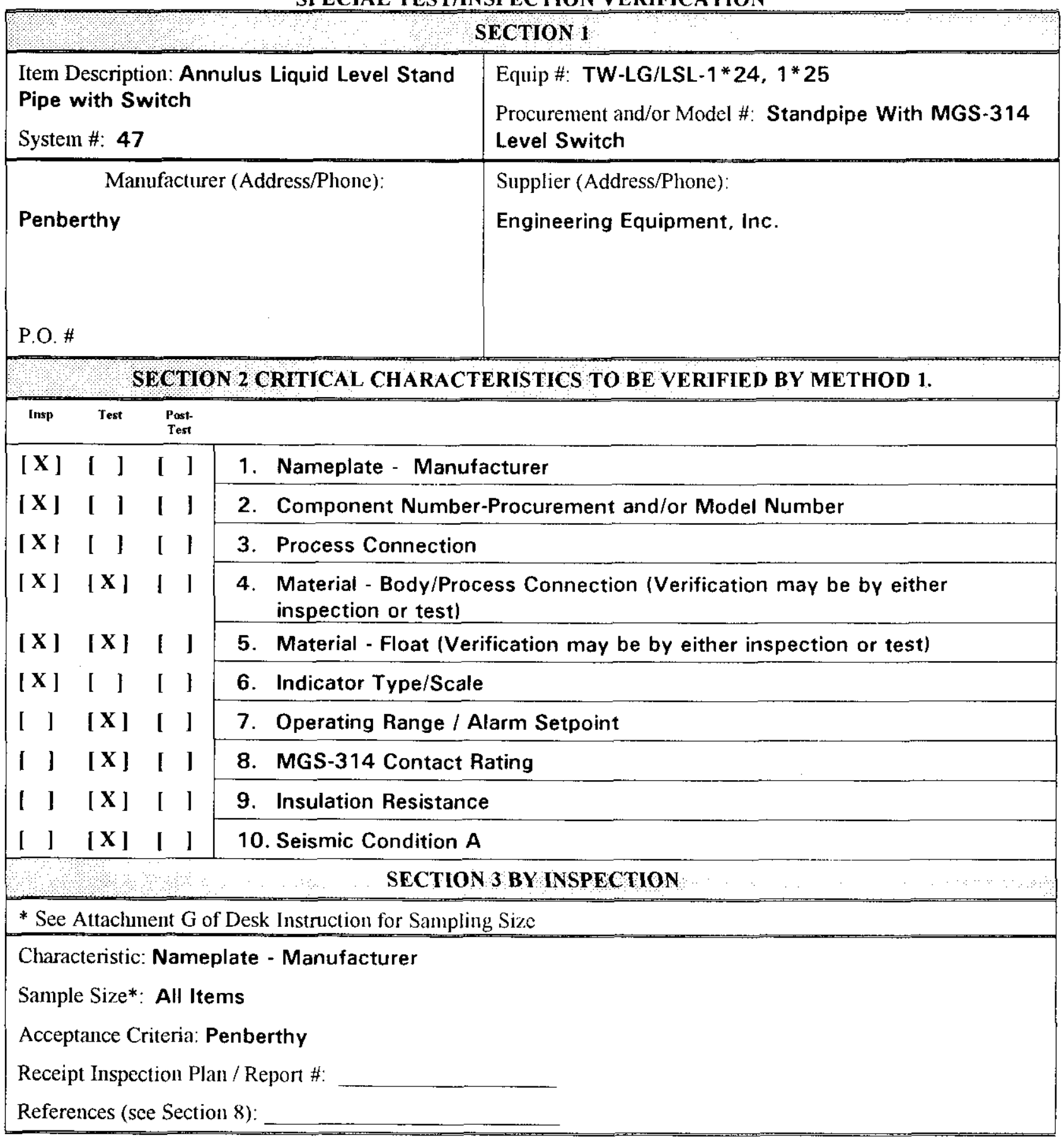




\begin{tabular}{|l|l|}
\hline Commercial Grade Item Upgrade Dedication Form & SN1-3882. Re1. 2 \\
\hline ECN No. $\frac{\text { NA }}{\text { CGI No. CGI-SNF-D-47-P4-007 }}$ & Page 8 o 12 \\
Title: CASK/MCO ANNULUS LIOUID LEVEL GAUGE/LEVEL SWITCH & \\
LOW & \\
\hline
\end{tabular}
Characteristic: Component Number-Procurement and/or Model Number
Sample Size*: All Items
Acceptance Criteria: Stand Pipe - 7A174-020; Level Switch - MGS-314. (Per Procurement Package W-441-P4, Rev. 2, Section G, Design Data Sheet)

Receipt Inspection Plan / Report \#:

References (see Section 8): Penberthy Multiview Manual, Application Report 4771

Characteristic: Process Connection

Sample Size*: All Items

Acceptance Criteria: 1" Flange

Receipt Inspection Plan / Report \#:

References (see Section 8)

Characteristic: Indicator Type/Scale

Sample Size*: All Items

Acceptance Criteria: Follower Type/Scale in inches of water (0-12 inches)

Receipt Inspection Plan / Report \#:

References (see Section 8):

Characteristic: Material - Body /Process Connection

Sample Size*: Normal Sampling Size

Acceptance Criteria: Stainless Steel

Receipt Inspection Plan / Report \#:

References (see Section 8):

Characteristic: Material - Float

Sample Size*: Normal Sampling Size

Acceptance Criteria: Stainless Steel

Receipt Inspection Plan / Report \#:

References (see Section 8): 


\begin{tabular}{l|l|}
\hline Commercial Grade Item Upgrade Dedication Form & SNF-3882. Rev. 2 \\
\hline ECN No. NA & CGI No. CGI-SNF-D-47-P4-007 \\
Titte: CASK/MCO ANNULUS LIOUID LEVEL GAUGE/LEVEL SWITCH & \\
\hline
\end{tabular}

\section{SECTION 4 B Y SPECIAL TEST}

* See Attachment $\mathrm{G}$ of Desk Instruction for Sampling Size

Test To Be Performed by:

[ ] Purchaser

I ] Supplier/Manufacturer**

Number of liems to be Tested:

Test/Inspection Location:

[ ] Other

Characteristic for Test: Operating Range / Alarm Setpoint

Acceptance Criteria: Provide water supply connection to upper/lower gage flanges, fill gage, set low level alarm trip at 6 inches, test switch at decreasing levels, alarm at 6 inches $+1-1 / 2$ inch.

Sample Size*: Normal Sampling Size

Actual Test Value:

Test Plan and Report \#: References (sec Section 8):

Characteristic for Test: MGS-314 Contact Rating

Acceptance Criteria: Nominal 5 AMP @ 120 VAC, Less Than 0.5 V Drop Across Contacts

Sample Size*: Normal Sampling Size

Actual Test Value:

Test Plan and Report \#: References (see Section 8):

Characteristic for Test: Insulation Resistance

Acceptance Criteria: Greater Than 10 Megohm Resistance To Ground At 1000v.

Sample Size*: Normal Sampling Size

Actual Test Value:

Test Plan and Report \#: References (sce Scction 8):

Characteristic for Test: Seismic Condition A, (TW-LG-1*24 and TW-LG-1*25 only)

Acceptance Criteria: 1. Maintain critical function (provide valid water level indication) before and after Seismic event. W-441-P4, Rev. 2, Appendix L, page L-10, provides a seismic testirig plan for these components at a (TBD) seismic spectra. After a seismic event, the level gauge follower may need to be reset with a handheld magnet.

Sample Size*: Destructively Test Only One Item

Actual Test Value:

Test Plan and Report \#: References (see Section 8):

**If Supplier/Manufacturer or Other, Refer to C(id Clecklist-2 for Suppont Information 


\begin{tabular}{|c|c|}
\hline Commercial Grade Item Upgrade Dedication Form & SNF-3882, Rev. 2 \\
\hline $\begin{array}{l}\text { ECN No. NA } \\
\text { CGI No. CGI-SNF-D-47-P4-007 } \\
\text { Title: CASK/MCO ANNULUS LIQUID LEVEL GAUGE/LEVEL SWITCH } \\
\text { LOW }\end{array}$ & Page 10 of 12 \\
\hline
\end{tabular}

Section 5 Test/ inspection Summan (Acceptance Method /)

\section{SUMMARY OF VERIFIED CRITICAL CHARACTERISTICS, THEIR VERIFICATION METHODS, AND RESULTS}

\section{ITEM DESCRIPTION:}

\begin{tabular}{|c|c|c|c|c|c|c|c|c|c|c|c|}
\hline \multicolumn{4}{|c|}{ Critical Characteristics } & \multicolumn{8}{|c|}{ Verification Results } \\
\hline Critical Characteristics & Acceptance Criteria Tolerances & ID & Function & $\begin{array}{l}\text { Method } \\
\text { T TIN }\end{array}$ & $\begin{array}{c}\text { Procedure } \\
\text { or RR }{ }^{\prime}\end{array}$ & $\begin{array}{l}\text { Check- } \\
\text { list ID }\end{array}$ & $\begin{array}{c}\text { Number } \\
\text { Tested }\end{array}$ & $\begin{array}{c}\text { Number } \\
\text { Failed }\end{array}$ & $\begin{array}{c}\text { Verifying } \\
\text { Organization }\end{array}$ & Printed Name Signature & Date \\
\hline $\begin{array}{l}\text { Nameplate - } \\
\text { Manufacturer }\end{array}$ & Penberthy & $\mathbf{X}$ & & & & & & & & & \\
\hline $\begin{array}{l}\text { Component Number- } \\
\text { Procurement Number } \\
\text { and/or Model Number }\end{array}$ & $\begin{array}{l}\text { Stand Pipe - 7A174- } \\
\text { 020; Level Switch - } \\
\text { MGS-314. (Per } \\
\text { Procurement Package } \\
\text { W-441-P4, Rev. 2, } \\
\text { Section G, Design Data } \\
\text { Sheet). }\end{array}$ & $\mathbf{X}$ & & & & & & & & & \\
\hline Process Connection & 1"Flange & $\mathbf{X}$ & & & & & & & & & \\
\hline $\begin{array}{l}\text { Material - Body/Process } \\
\text { Connection }\end{array}$ & Stainless Steel & $\mathbf{X}$ & & & & & & & & & \\
\hline Material - Float & Stainless Steel & $\mathbf{X}$ & & & & & & & & & \\
\hline Indicator Type/Scale & $\begin{array}{l}\text { Follower Type/Scale in } \\
\text { inches of water }(0-12 \\
\text { inches! }\end{array}$ & $\mathbf{X}$ & & & & & & & & & \\
\hline $\begin{array}{l}\text { Operating Range / Alarm } \\
\text { Setpoint }\end{array}$ & $\begin{array}{l}\text { Provide water supply } \\
\text { connection to } \\
\text { upper/lower gage } \\
\text { flanges, fill gage, set } \\
\text { low level alarm trip at } 6 \\
\text { inches, test switch at } \\
\text { decreasing levels, alarm } \\
\text { at } 6 \text { inches }+1-1 / 2 \\
\text { inch. }\end{array}$ & & $\mathbf{X}$ & & & . & & & & . & \\
\hline $\begin{array}{l}\text { Level Switch MGS-314 } \\
\text { Contact Rating }\end{array}$ & $\begin{array}{l}\text { Nominal } 5 \text { AMP @ } 120 \\
\text { VAC, Less Than } 0.5 \mathrm{~V} \\
\text { Drop Across Contacts }\end{array}$ & & $\mathbf{X}$ & & & & & & & & \\
\hline
\end{tabular}


Commercial Grade Item Upgrade Dedication Form

ECN No. NA CGI No. CGI-SNF-D-47-P4-007

Title: CASK/MCO ANNULUS LIQUID LEVEL GAUGE/LEVEL SWITCH

LOW

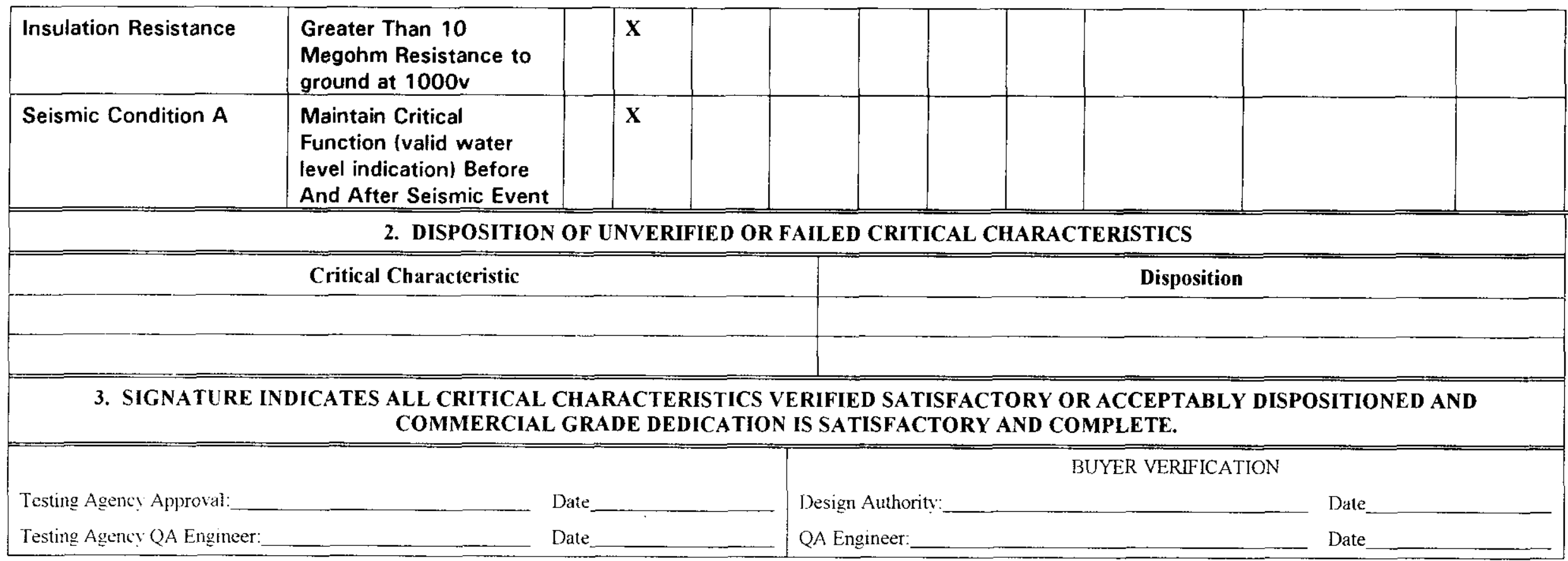


Commercial Grade Item Upgrade Dedication Form

ECN No. NA CGI No. CGI-SNF-D-47-P4-007

Title: CASK/MCO ANNULUS LIOUID LEVEL GAUGE/LEVEL SWITCH

LOW
SNF-3882, Rev 2

Page 12 of 12

\begin{tabular}{|c|c|c|}
\hline \multicolumn{3}{|c|}{ Section 6 Contacts/Phone Numbers } \\
\hline \multicolumn{2}{|l|}{ Name } & Phone \\
\hline \multicolumn{3}{|l|}{ Design Authority } \\
\hline \multicolumn{3}{|l|}{ QA } \\
\hline \multicolumn{3}{|l|}{$\mathrm{QC}$} \\
\hline \multicolumn{3}{|l|}{$\begin{array}{l}\text { Cog - Engineer } \\
\text { CGI Enoineer }\end{array}$} \\
\hline \multicolumn{3}{|l|}{ CGI Engineer } \\
\hline \multicolumn{3}{|l|}{ Procurement Engineer } \\
\hline \multicolumn{3}{|l|}{ Other } \\
\hline Section 7 Suppoiting Do & nentation for this Checkit & \\
\hline Initial Procurement Documents & For Critical Charac & ristics \\
\hline \multicolumn{3}{|l|}{ I J Drawings: } \\
\hline \multicolumn{3}{|l|}{ I I Manuals (specify type \& number): } \\
\hline \multicolumn{3}{|l|}{ I I Design Calculations } \\
\hline \multicolumn{3}{|l|}{ [ ] Installation Instructions } \\
\hline \multicolumn{3}{|l|}{ I I Operation Instructions } \\
\hline \multicolumn{3}{|l|}{ I ] Calibration Instructions } \\
\hline \multicolumn{3}{|l|}{ I ] Manufacturer's Recommended Spare Parts Lisı } \\
\hline \multicolumn{3}{|l|}{ [ ] Other: } \\
\hline \multirow{2}{*}{\multicolumn{3}{|c|}{ Procurement Documents }} \\
\hline I I Certificate of Conformance/Compliance & & \\
\hline \multicolumn{3}{|l|}{ I ] Seismic Qualification Certificate } \\
\hline \multicolumn{3}{|l|}{ I I Environmental Qualification Certificate } \\
\hline \multicolumn{3}{|l|}{ I J Test Report (s): } \\
\hline \multicolumn{3}{|l|}{ I I Inspection Report (s): } \\
\hline \multicolumn{3}{|l|}{ I J CMTRs for ASME Pressure Retaining Materials } \\
\hline \multicolumn{3}{|l|}{ I I Valve Seat Leakage Report } \\
\hline \multicolumn{3}{|l|}{ 1 ] Weld Records } \\
\hline \multicolumn{3}{|l|}{ I I Material Traceability Record } \\
\hline I ] Other: & & \\
\hline
\end{tabular}




\section{DISTRIBUTION SHEET}

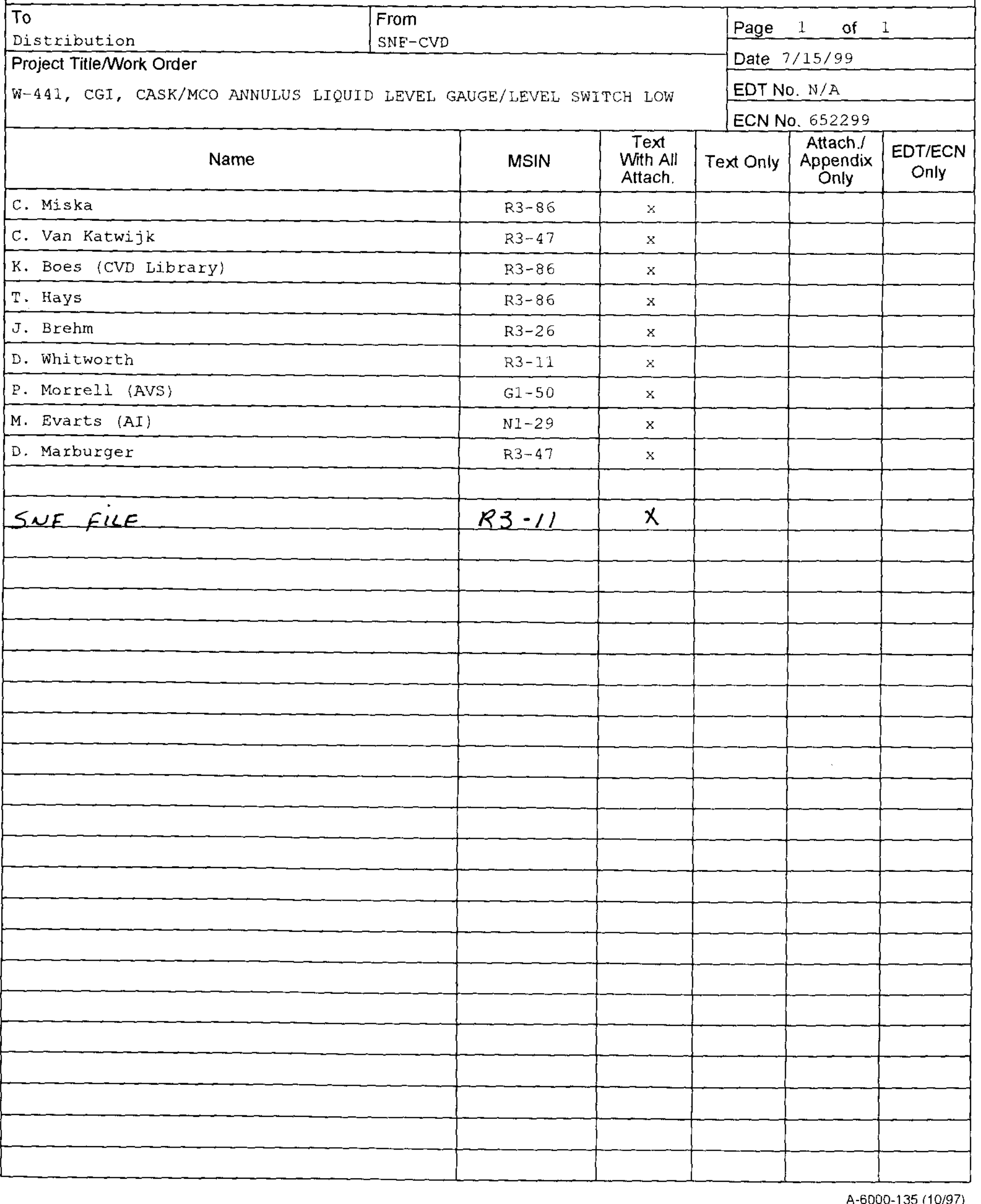

